\title{
Prevalence of Congenitally Missing Lateral Incisors and Peg Laterals in Patients Receiving Orthodontic Treatment
}

\author{
Dr Radha Baral,' Dr Dipshikha Bajracharya,, Dr Bidhata Ojha,3 Dr Ganesh Silwal4 \\ .3Lecturer, Department of Oral Pathology, ${ }^{2}$ Associate Professor, ${ }^{4}$ Private Practitioner \\ Kantipur Dental College, Kathmandu, Nepal
}

Correspondence: Dr Radha Baral; Email: radhabarall22@gmail.com

\section{ABSTRACT}

Introduction: Maxillary lateral incisor is a common congenitally missing tooth in the oral cavity. Maxillary lateral incisor is also the most common varying tooth in form than any other tooth. The objective of the study is to estimate the prevalence of congenitally missing maxillary lateral incisors and peg laterals in patients receiving orthodontic treatment.

Materials \& Method: This is a cross sectional retrospective study carried out among 204 orthodontic patients. Panoramic radiographs taken during the diagnostic procedure of orthodontic treatment was used to confirm the presence or absence of lateral incisors. The presence of peg laterals was determined by dental casts.

Result: Prevalence of missing maxillary lateral incisors and peg laterals was found $2.5 \%$ and $3.9 \%$ respectively. Prevalence of missing lateral in male was $5.7 \%$ while among female no missing laterals were observed. Prevalence of peg laterals in male and female was $1.1 \%$ and $6 \%$ respectively. Among the missing lateral incisors, prevalence of bilaterally missing lateral incisors was $60 \%$ while prevalence of both right and left unilateral missing laterals was $20 \%$. Among the peg laterals, bilateral peg laterals were present in $50 \%$ patients, followed by left unilateral $37.5 \%$ and right unilateral $12.5 \%$ peg laterals.

Conclusion: The present study shows higher prevalence of peg laterals than missing lateral incisors. Prevalence of missing lateral incisors was higher in male and prevalence of peg laterals was higher in female. Prevalence of bilaterally missing laterals and bilateral peg laterals was higher than unilateral presentation.

Keywords: Maxillary lateral incisor, Peg lateral, Prevalence.

\section{INTRODUCTION}

Agenesis of teeth is one of the most common of human developmental anomalies.' A tooth is defined as congenitally missing if it has not erupted in the oral cavity and is not visible radiographically. ${ }^{2}$ Although any tooth can be susceptible to agenesis, maxillary lateral incisors and second premolars show a great possibility of agenesis. ${ }^{3}$ Maxillary lateral incisor is also the most common varying tooth in form than any other tooth in the oral cavity after third molars. ${ }^{3}$ A common situation is to find maxillary lateral incisors with nondescript, pointed form; such teeth are called peg-shaped laterals or peg laterals. ${ }^{4}$

According to Moyers there are five principal known causes of congenital absence of teeth. Heredity, ectodermal dysplasia, conditions such as rickets, syphilis and expression of evolutionary changes in the dentition. ${ }^{4.5}$ Face and jaws tend to decrease in the anteroposterior direction with the evolution of species. This trend can limit the space needed to accommodate all of the teeth and, consequently, the last tooth of each series tends to disappear (third molars, second premolars and lateral incisors). This is a hereditary process: a generation that has an anomalous tooth (small sized maxillary lateral incisors/ peg-shaped lateral incisors) will have descendants that no longer possess this tooth. ${ }^{5}$ Tooth agenesis may occur in the form of an isolated familial or sporadic anomaly or in association with other genetic diseases like Cleft lip/palate, Down syndrome, Van der Woude syndrome, Ectodermal dysplasia, Hypohidrotic dysplasia, Incontinenita pigment, Witkop syndrome, Rieger syndrome, Holoprosencephaly. In nonsyndromic agenesis, gene mutations are said to be the cause. The mutations in genes responsible for tooth development are marked as PAX9 and MSX1.6-8

There is remarkably little information in the literature on the prevalence of missing teeth and variation in shape of teeth in Nepalese context. The aim of the study was to investigate the prevalence of the congenitally missing 
lateral incisors and peg laterals in patients receiving orthodontic treatment.

\section{MATERIALS AND METHOD}

This is a cross sectional retrospective study conducted in Kantipur Dental College, Basundhara, Kathmandu, Nepal among the patients receiving orthodontic treatment. The duration of the study was from May 2019 to July 2019. Ethical clearance for the study was taken from the institutional review committee (IRC) of Kantipur Dental College.

Sample size was calculated using following formula,

Required sample size $(n)=\frac{Z a^{2} P Q}{d^{2}}$

Where,

$\mathrm{Za}=1.96$ for $95 \%$ reliability

According to Sharma $\mathrm{JN}^{9}$ estimated prevalence $(\mathrm{P})=$ $12.6 \%$

$Q=100-12.6=87.4 \%$

$d=$ Maximum tolerable error $=5 \%$

Applying all the values in the mentioned formula sample size is 170 . Amplifying the sample size by $10 \%$ for processing errors and another $10 \%$ for non-response errors, the final sample size becomes $170+17+17=204$

Patients between 10 years to 40 years of age receiving orthodontic treatment were included in the study. Patients with cleft lip and palate, hypodontia or oligodontia as a part of syndrome, patient with missing of lateral incisors because of extraction or avulsion or any other cause were excluded from the study.
Panoramic radiographs taken during the diagnostic procedure of orthodontic treatment was used to confirm the presence or absence of lateral incisors. The presence of peg laterals was determined by dental casts. Dental history sheets were used to rule out extractions and avulsions as well as presence of any syndrome or cleft lip and palate. Data analysis was done using SPSS version 21. Qualitative data was presented as frequencies and percentages. Quantitative data was presented as means and standard deviation. Chi square test was used to show the association.

\section{RESULT}

Total sample 204 was evaluated, among which 87(42.6\%) were male and $117(57.4 \%)$ were female. Mean age of the patient was $18.51 \pm 5.67$ years. We also categorized the patient according to the ethnic group present in Nepal. ${ }^{10}$ Distribution of the patient according to the ethnic group present in Nepal shows that highest number of patients $61.8 \%(126)$ belonged to Brahamin/Chhetri followed byJanjati which includes $22.5 \%(46), 9.3 \%(19)$ patients belonged to Newar community and $5.4 \%(11)$ of patients belonged to Tarai/Madhesi. Least number of patient 1\%(2) belonged to Muslim community(Figure 1).

Prevalence of missing maxillary lateral incisors and peg laterals was $2.5 \%(5)$ and $3.9 \%$ (8) respectively (Figure 2 and Figure 3) which shows that prevalence of peg laterals in orthodontic patients was higher than missing lateral incisors.

Distribution of the missing laterals and peg laterals according to the site is shown in Figure 4 and Figure 5. Among the missing lateral incisors, prevalence of bilaterally missing lateral was $60 \%$ (3) while prevalence of both right

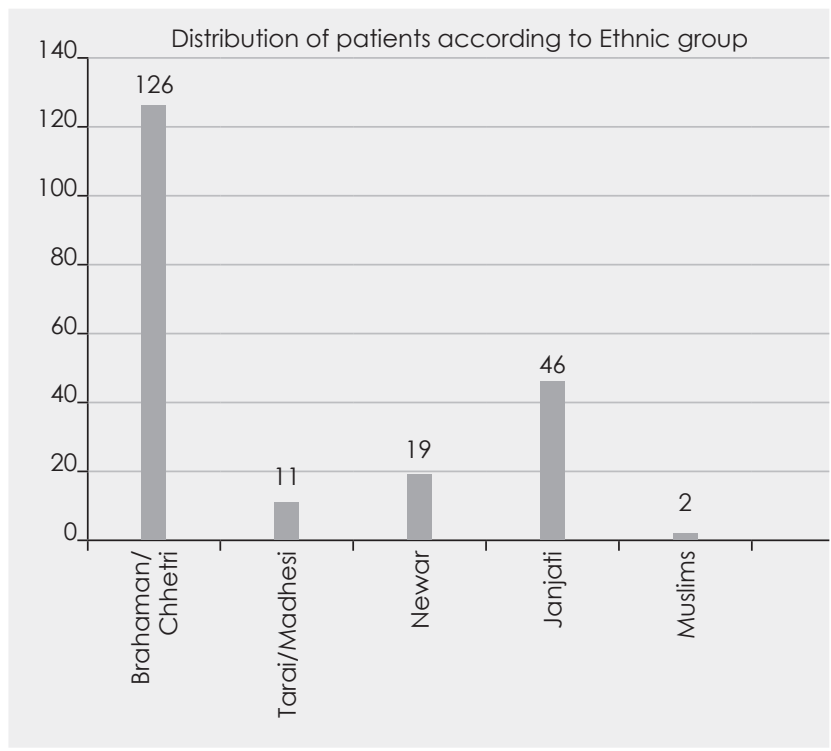

Figure 1: Distribution of patient according to the different ethnic group 


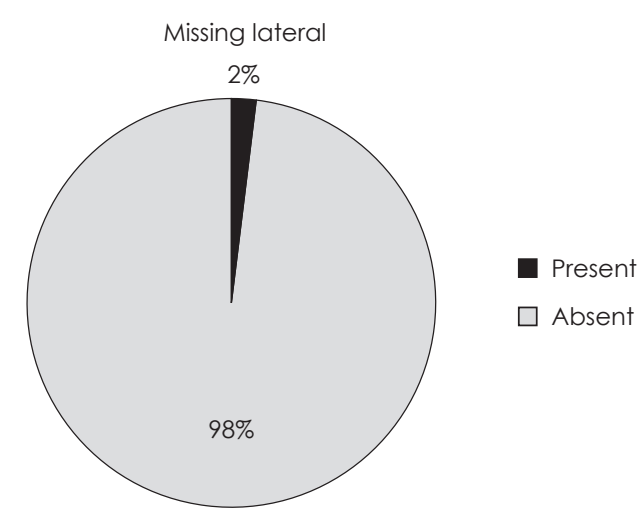

Figure 2: Frequency of missing laterals

Sites specificity of Missing lateral incisors

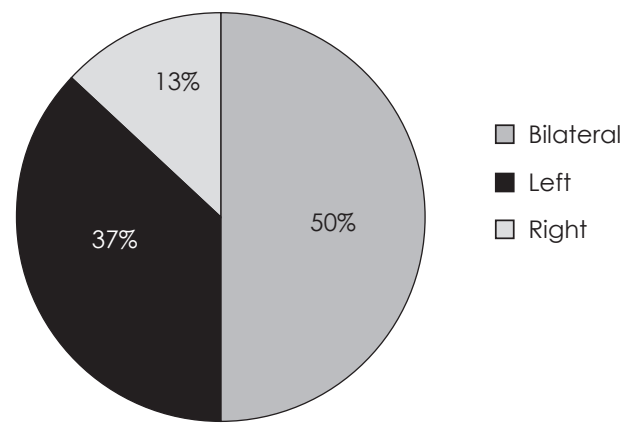

Figure 4: Site specificity of missing lateral

and left unilateral missing was $20 \%$ (1). Among the total peg laterals observed, bilateral peg laterals were present in $50 \%$ (4) which was followed by left unilateral $37.5 \%$ (3) and right unilateral $12.5 \%$ (1) peg laterals.

Prevalence of missing lateral in male was $5.7 \%$ (5) while among female no missing laterals were observed. Prevalence of missing lateral incisors was higher in male which was statistically significant $(p<0.05)$. Prevalence of peg laterals in male and female was $1.1 \%$ (1) and 6\% (7) respectively. Prevalence of peg laterals was higher in female which was statistically significant $(p<0.05)$. Among the patients with missing laterals $100 \%$ (5) were male and among the patients of peg laterals $25 \%$ (2) were male and $75 \%$ (6) were female. Furthermore, $40 \%$ (2) of missing lateral cases and $12.5 \%(1)$ peg lateral cases were associated with other missing teeth.

\section{DISCUSSION}

Agenesis of tooth results from disturbances during the early stages of tooth development. ${ }^{2}$ Aberrations in tooth morphology resulting from late disturbances during the differentiation process most commonly result in size variations. ${ }^{4}$ Tooth agenesis is the most common developmental anomaly of human dentition, occurring in approximately $25 \%$ of the population. The third molar is the most affected tooth, showing a prevalence of $20.7 \% .^{5}$ Maxillary lateral incisor is the second most frequently missing tooth, followed by the

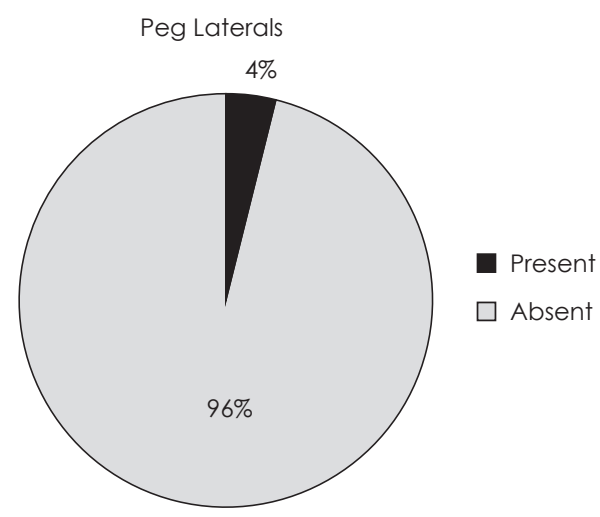

Figure 3: Frequency of Peg laterals

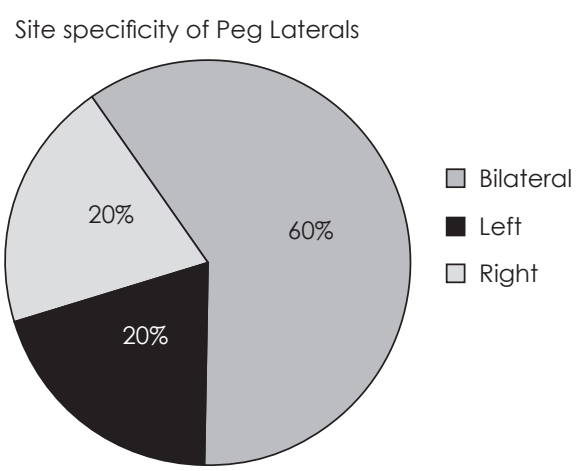

Figure 5: Site specificity of Peg laterals

mandibular second premolar, maxillary second premolar and mandibular incisor."

In the present study prevalence of missing laterals was $2.5 \%$ (5) (Figure 2). Prevalence among male was $5.7 \%$ (5) while prevalence among female was 0 . All the patients having missing lateral incisors were male. Frequency of the missing lateral incisors has been found $0.3 \%$ to $11.3 \%$ in different populations. ${ }^{3}$ In a study done in Portuguese population prevalence of missing lateral incisors was found $1.3 \%{ }^{2}$ The prevalence for missing maxillary lateral incisors was $1.91 \%$ in dental patients in study done by Arandi NZ et al. ${ }^{7}$ In the same study prevalenceof missing lateral incisors in males was $1.7 \%$ and in females was $2.1 \%$ which is in contrast with our study. In a similar study done in orthodontic patients prevalence of missing lateral incisor was $9.42 \% .{ }^{3}$ In contrast to our findings, of patients with agenesis, $44.12 \%$ were female and $55.88 \%$ were male. $^{3}$ In another study done in Iranian population the prevalence of missing maxillary lateral incisor was $18.94 \% .^{12}$ Prevalence of missing teeth was found in $12.6 \%$ of the patient receiving orthodontic treatment in a tertiary care center of Nepal. Among the missing teeth maxillary lateral incisors were found to be the most commonly missing tooth excluding third molars. ${ }^{9}$ These finding demonstrate there is great variation in prevalence of missing lateral in incisors in different population.

Prevalence of bilateral missing laterals was $60 \%$ (3) while prevalence of both right and left unilateral missing was $20 \%$ (1). Similar to present study, Afzal Z et al. observed $44.12 \%$ 
bilateral, $23.53 \%$ right unilateral and $32.35 \%$ left unilateral expression of missing laterals in orthodontic patients. ${ }^{3}$ Similar finding was seen in other studies showing higher bilateral absence of lateral incisors than unilateral. ${ }^{2,4,12}$ These findings suggest that that bilateral missing lateral is more common than unilateral expression.

In the present study the prevalence of peg lateral in orthodontic patient was found $3.9 \%$ (8) (Figure3). Among the patients having peg laterals $25 \%(2)$ were male and $6(75 \%)$ were female. In similar studies done on orthodontic patients the prevalence of peg laterals was found $1.3 \%$ and $5.26 \%$ respectively. ${ }^{3,4}$ In a systematic meta-analysis of prevalence of the peg lateral incisors, prevalence in general population, dental patients and orthodontic patients were pooled at 1.6\%, $1.9 \%$ and $2.7 \%$ respectively. ${ }^{13}$ These studies also demonstrate higher prevalence in female compared to male which was also observed in our study. ${ }^{3,4,13}$ In the present study, among the total peg laterals observed bilateral peg laterals was present in $50 \%$ (4) which was followed by left unilateral $37.5 \%$ (3) and right unilateral $12.5 \%(1)$ peg lateral. Similar to these findings other studies also show higher prevalence of bilateral peg lateral followed by unilateral left and right peg lateral. ${ }^{3,4}$

Congenitally missing lateral incisors whether unilateral or bilateral may affect the self-esteem and social relationships of the affected individual along with some esthetic and functional problems. Absence of maxillary lateral incisors may also cause a diastema between the central incisors, spacing between permanent incisor and canine, mesial migration of canines, midline shift in case of a unilateral missing tooth. ${ }^{6}$ Similarly peg laterals also pose esthetic and functional problems. Early recognition of a tooth agenesis is helpful in order to provide adequate treatment and prevent a developing malocclusion. ${ }^{4}$ Treatment of missing lateral and peg laterals can include orthodontic space opening or closure before prosthetic therapy, using adhesive restorative techniques, removable or fixed partial dentures, implant supported restorations, or combinations of this approaches.'

The data in this study is from a single institution so it may have some limitations. Hence a multi-centric data with larger sample size may overcome this limitation.

\section{CONCLUSION}

The present study shows higher prevalence of peg laterals than missing lateral incisors. Prevalence of missing lateral incisor was higher in male. Prevalence of peg lateral was higher in female. Prevalence of bilaterally missing laterals and bilateral peg laterals was higher than unilateral presentation.

\section{ACKNOWLEDGEMENT}

We would like to acknowledge Dr Sanzee Silwal, Dr Puja Shah, Dr Asal Acharya, Dr Anju Agrawal, Dr Diwash Adhikari and Dr Ravi Ranjan Kumar Rajak for their contributions in data collection.

\section{OJN}

\section{REFERENCES}

1. Bural C, Oztas E, Ozturk S, Bayraktar G. Multidisciplinary treatment of non- syndromic oligodontia. Eur J Dent. 2012;6(2):218-26.

2. Pinho T, Tavares P, Maciel P, Pollmann C. Developmental absence of maxillary lateral incisors in the Portuguese population. Eur J Orthod. 2005;27(5):443-9.

3. Afzal F, Rasool G, Bashir S, Afzal S, Gul H. Prevalence of Congenitally Missing Maxillary Lateral Incisor and Peg Laterals in a Local Orthodontic Population. JKCD. 2015;5(2):24-7.

4. Amin F, Asif J, Akber S. Prevalence of peg laterals and small size lateral incisors in orthodontic patients - A study. Pakistan Oral Dent J. $2011 ; 31(1): 88-91$.

5. Almeida RR, Morandini AC, Almeida-Pedrin RR, Cristina RC, Cristina FR, Insabralde NM. A multidisciplinary treatment of congenitally missing maxillary lateral incisors : A 14-year follow-up case report. J Appl oral Sci. 2014;22(5):465-71.

6. Creton MA, Cune MS, Verhoeven JW, Meijer GJ. Patterns of Missing Teeth in a Population of Oligodontia Patients. Int J Prosthodont. 2007;20(4):409-13.

7. Arandi NZ, Mustafa S. Maxillary lateral incisor agenesis; A retrospective cross-sectional study. Saudi Dent J. King Saud University; 2018;30(2):155-60.

8. Jana S, Chakraborty A, Dey B, Ghosh C, Roy D. Congenitally Missing Lateral Incisor in Primary Dentition - A Report of Two Cases. Int J Oral Heal Med Res. 2016;3(2):29-31.

9. Sharma JN. Pattern of distribution of malocclusions in patients seeking orthodontic treatment at bpkihs from sunsari district of nepal. Heal Renaiss. 2010;8(2):93-6.

10. Bennett L, Dahal DR, Govindasamy P. Caste ethnic and regional identity in Nepal: Further analysis of the 2006 Nepal Demographic and Health Survey. Calverton Maryland Macro International; 2008.

11. Sisman Y, Uysal T, Gelgor IE. Hypodontia. Does the Prevalence and Distribution Pattern Differ in Orthodontic Patients? Eur J Dent. 2007;1 (3):167-73.

12. Sheikhi M, Sadeghi MA, Ghorbanizadeh S. Prevalence of congenitally missing permanent teeth in Iran. Dent Res J (Isfahan). $2012 ; 9$ (1):105.

13. Hua F, He H, Ngan P, Bouzid W. Prevalence of peg-shaped maxillary permanent lateral incisors: A meta-analysis. Am J Orthod Dentofac Orthop. 2013;144(1):97-109. 